\title{
Hippocampal Sclerosis in Dementia, Epilepsy, and Ischemic Injury: Differential Vulnerability of Hippocampal Subfields
}

\author{
Kimmo J. Hatanpaa, MD, PhD, Jack M. Raisanen, MD, Emily Herndon, MD, Dennis K. \\ Burns, MD, Chan Foong, MS, Amyn A. Habib, MD, and Charles L. White III, MD \\ Alzheimer's Disease Center (KJH, EH, CF, CLW) and Department of Pathology $(\mathrm{KJH}, \mathrm{JMR}, \mathrm{EH}$, \\ DKB, CF, CLW), and Neurology and Neurotherapeutics (AH), University of Texas Southwestern \\ Medical Center, Dallas, Texas; and VA North Texas Health Care System, Dallas, Texas (AH)
}

\section{Abstract}

Severe neuronal loss in the hippocampus, that is, hippocampal sclerosis (HS), can be seen in 3 main clinical contexts: dementia (particularly frontotemporal lobar degeneration [FTLD]), temporal lobe epilepsy (TLE), and hippocampal ischemic injury (H-I). It has been suggested that shared pathogenetic mechanisms may underlie selective vulnerability of the hippocampal subfields such as the CA1 in these conditions. We determined the extent of neuronal loss in cases of HS-FTLD ( $n=14)$, HS-TLE $(n=35)$, and H-I $(n=20)$. Immunohistochemistry for zinc transporter 3 was used to help define the $\mathrm{CA} 3 / \mathrm{CA} 2$ border in the routinely processed human autopsy tissue samples. The subiculum was involved in 57\% of HS-FTLD, $10 \%$ of $\mathrm{H}-\mathrm{I}$, and $0 \%$ of HS-TLE cases ( $\mathrm{p}<0.0001)$. The CA regions other than CA1 were involved in 57\% of HS-TLE, $30 \%$ of $\mathrm{H}-\mathrm{I}$, and $0 \%$ of HS-FTLD cases ( $\mathrm{p}=0.0003$ ). The distal third of CA1 was involved in $79 \%$ of HS-FTLD, 35\% of H-I, and 37\% of HS-TLE cases ( $\mathrm{p}=0.02)$. The distal third of CA1 was the only area involved in $29 \%$ of HS-FTLD, $3 \%$ of HS-TLE, and $0 \%$ of H-I cases $(\mathrm{p}=0.019)$. The proximal-middle CA1 was the only area affected in 50\% of H-I, 29\% of HS-TLE, and 0\% of HSFTLD cases $(p=0.004)$. These findings support heterogeneity in the pathogenesis of HS.

\section{Keywords}

Brain ischemia; Frontotemporal dementia; Frontotemporal lobar degeneration; Hippocampal sclerosis; Mesial temporal sclerosis; Vascular dementia; ZNT3

\section{INTRODUCTION}

The histopathologic finding of hippocampal sclerosis (HS) can be seen in association with dementia (particularly frontotemporal lobar degeneration [FTLD]), temporal lobe epilepsy (TLE), and hippocampal ischemic injury (H-I). The CA1 subregion of the hippocampus is selectively vulnerable to HS regardless of the etiology; other subregions can also be affected. For example, the CA4 subregion is involved in a subset of TLE cases (1-3), but

Copyright (ㅇ 2014 by the American Association of Neuropathologists, Inc.

Send correspondence and reprint requests to: Kimmo J. Hatanpaa, MD, PhD, Department of Pathology, University of Texas Southwestern Medical Center, 5323 Harry Hines Blvd, Dallas, Texas 75390-9073; Kimmo.Hatanpaa@UTSouthwestern.edu. 
involvement of this subregion is relatively uncommon in FTLD and $\mathrm{H}-\mathrm{I}$ (4). However, it is unclear to what extent the anatomic distribution of HS can help in the differential diagnosis of the underlying condition in autopsy neuropathology. In this study, our first goal was to address this question by directly comparing the distribution of HS in cases of FTLD, TLE, and $\mathrm{H}-\mathrm{I}$. Our second goal was to contribute to the understanding of the pathogenesis of HS. If the distribution of HS is different the 3 conditions, this would argue that the pathogenetic pathways leading to HS do not converge on common processes such as excitoxicity, proteasomal dysfunction, and ischemia in these conditions.

Autopsy studies have reported the prevalence of HS in the elderly to range between $0.4 \%$ and $26 \%$ (5). The wide range in the reported prevalence is likely caused by factors such as the age of the population studied, precise histopathologic definition of HS, and whether neuronal loss in the context of Alzheimer disease (AD) is also considered HS. Alzheimer disease is almost always associated with some loss of hippocampal pyramidal neurons, and, rarely, hippocampal neuronal loss in AD can be severe and resemble HS. Severe neuronal loss in AD is typically associated with frequent "ghost" or extracellular neurofibrillary tangles, which suggests that the neurons died secondary to the AD pathology. Whether neuronal loss in such AD cases should be considered to represent combined AD and HS or simply AD is largely a semantic issue. In this study, we excluded cases with significant AD pathology. Furthermore, we elected to define HS as very severe, near-total loss of neurons involving a part or all of any of the hippocampal subfields in the clinical context of either dementia or TLE.

Ischemic injury to the brain often results in neuronal loss in the hippocampus, which is selectively vulnerable to ischemia. Histologically, the earliest finding is acute anoxicischemic injury (red neuron change). Later, $\mathrm{H}-\mathrm{I}$ may result in 2 types of histologic alterations. In the first type, $\mathrm{H}-\mathrm{I}$ takes the form of a classic microinfarct with cavitation, foamy macrophages, and prominent capillaries. If the ischemic injury is more insidious, a second type of histologic alteration results, consisting of neuronal dropout without cavitation and with a histologic picture similar to HS-FTLD and HS-TLE $(4,6)$.

In large longitudinal studies, HS in the elderly was not associated with an increased incidence of systemic cardiovascular risk factors such as hypertension, transient ischemic attacks, or smoking (5). However, depending on the study, HS showed a weak association ( $p$ $<0.05$ ) with either large or lacunar infarcts elsewhere in the brain (5), leaving open the possibility that ischemic injury may contribute to the pathogenesis of HS in some elderly patients.

Although the mechanisms underlying the selective vulnerability of hippocampal neurons are not completely understood, there are 2 established hypotheses that are worth mentioning. In hypoxic-ischemic injury, there is strong evidence supporting a role for glutamate excitotoxicity in the selective vulnerability of the CA1 subregion over other subregions such as the CA3. This evidence includes hippocampal organotypic slice culture studies showing that CA1 neuronal death is prevented by NMDA and AMPA receptor antagonists, much larger intraneuronal increases in NMDA-induced $\mathrm{Ca}^{++}$are observed in CA1 compared with CA3 neurons (7), and there is a higher density of polyamine-sensitive NMDA receptors in 
CA1 compared with CA3 (8). Another hypothesis that might explain the vulnerability of the CA1 subregion not only in hypoxic-ischemic injury but also in neurodegenerative diseases argues that the CA1 is particularly vulnerable to impaired protein degradation by the proteasome. Proteasomal dysfunction is implicated in these conditions because of the observed accumulation of misfolded and ubiquitinated proteins in neurons (9). In hippocampal slice cultures, proteasomal inhibition results in a selective neuronal death in the CA1, and this neuronal death can be prevented by modulating apoptotic signal transduction pathways (10).

To our knowledge, this is the first study in which the anatomic extent of HS was directly compared among FTLD, TLE, and H-I cases in rigorously defined hippocampal subfields. Our novel findings include observations that the distal third of CA1 (CA1-dist) and prosubiculum are the areas most commonly involved in FTLD, whereas the proximal (CA1prox) and middle CA1 (CA1-mid) are more likely to be involved in TLE and H-I. We also demonstrate that labeling of the hippocampal mossy fibers by immunohistochemistry for ZNT3, a zinc transporter associated with zinc-containing synaptic vesicles, is helpful in defining the border between CA 3 and CA 2 in routinely processed formalin-fixed human autopsy tissue.

\section{MATERIALS AND METHODS}

\section{Cases}

The study included autopsy brains with HS-FTLD $(n=14$; all with TAR-DNA binding protein 43 [TDP-43] or fused in sarcoma inclusions, no significant AD pathology, and a history of dementia), HS-TLE ( $n=35$; with a history of TLE), and H-I ( $n=20$; either severe hippocampal neuronal loss with a clinical history of an ischemic episode or histologic evidence of ischemic neuronal injury). The median ages were 69.5 years (range, 47-99 years), 41 years (6-65 years), and 51 years (7-85 years) in the HS-FTLD, HS-TLE, and H-I groups, respectively. The tissue was obtained from the archives of the Division of Neuropathology, Department of Pathology and Brain Bank of the Alzheimer Disease Center, UT Southwestern Medical Center, Dallas, Tex. A letter was received from the UT Southwestern Medical Center Institutional Review Board stating that studies on autopsy tissue do not require an institutional review board approval.

The H-I group consisted of cases with a clinical history of an ischemic event such as cardiac arrest and the finding of severe hippocampal neuronal death at autopsy. Although hippocampal cavitary infarcts have not been considered to represent HS in most previous studies, we included H-I cases with either neuronal dropout alone or neuronal dropout with cavitation; the rationale for this was that both histologic patterns represent $\mathrm{H}-\mathrm{I}$ and provide information on the distribution of H-I. Microscopically, the H-I cases showed areas with frequent red neuron change (prominent cytoplasmic eosinophilia associated with nuclear pyknosis) or severe neuronal dropout that was histologically indistinguishable from HS (Fig. 1). Of note, some of the cases in the H-I group had hippocampal cavitation (i.e. infarcts) in addition to neuronal loss and, therefore, would not meet a definition of HS that excludes cavitation (11). An H-I case with necrosis involving the entire medial temporal lobe and all 
hippocampal subfields was excluded because it would not provide information on selectively vulnerable hippocampal subfields.

All of the HS-FTLD cases with TDP-43-positive inclusions had TDP-43-positive inclusions and neurites in the hippocampus. In the oldest 3 cases (aged 86, 86, and 99 years), the TDP-43 pathology was limited to the hippocampus; in the remaining 11 HS-FTLD cases, TDP-43 pathology was also present in the frontal neocortex. Recently, it has been suggested that HS in the oldest old with dementia represents a separate disease, that is, "HS-Aging" (5). The precise clinical and pathologic criteria that define HS-Aging and distinguish it from FTLD-TDP await elucidation by additional studies.

\section{Evaluation of HS and Ischemic Injury}

The extent of HS, defined as near-total loss of hippocampal neurons, was evaluated in hematoxylin and eosin (H\&E)-stained sections at the level of the lateral geniculate nucleus. Gliosis with only mild to moderate neuronal loss was not considered to be HS.

The CA1 region was divided into proximal (CA1-prox), middle (CA1-mid), and distal (CA1-dist) thirds arbitrarily, that is, the division was not based on anatomic landmarks. Rather, the CA1 region was divided into 3 roughly equal parts. The borders of CA1, CA2, CA3, CA4, and subiculum were outlined primarily based on H\&E histology $(12,13)$. The CA2 region is usually narrower and more densely populated than the CA3 region. The CA1 region is wider and more sparsely populated than the CA2 region. The subiculum proper is usually somewhat wider than CA1 and characterized by an undulating layer of neurons on the side facing the dentate gyrus; this layer breaks into separate islands of neurons in the presubiculum. The border between CA1 and subiculum is typically oblique.

Immunohistochemistry and special stains were used as supporting studies in individual cases.

The term "prosubiculum" was used to denote the proximal segment of the subiculum that overlaps with the CA1; our usage of the term is similar to that of Rosene and Van Hoesen (14) but differs from that of Lorente de No (15), who used this term to refer to a large segment that is currently considered to correspond to the subiculum proper (16). The justification for the term "prosubiculum" has been questioned by some authors who have maintained that prosubiculum is simply a part of the subiculum proper with no distinguishing features (16). Very recently, however, Ding (17) published a comprehensive study of the subicular complex, clearly demonstrating that prosubiculum is a separate subfield, with its unique neurochemical and cytoarchitectonic features, protein expression profile, and connections to the amygdala.

To our knowledge, there are no robust immunohistochemical markers or stains that would aid in defining the CA1/subiculum border and that would work consistently on routinely processed paraffin sections of the adult human autopsy brain. Enzyme histochemistry for acetylcholinesterase was recently reported to highlight the prosubiculum (the most proximal segment of the subiculum) in the monkey brain, but this stain requires frozen sections (17). However, the border between the CA1 and the subiculum (prosubiculum) can be readily identified on H\&E- or Nissl-stained sections in most cases (16). The CA1/subiculum 
boundary is typically characterized by a marked widening of the pyramidal cell layer. The boundary is oblique, with an angle that varies greatly from one brain to another. Sometimes, the oblique CA1/subiculum boundary corresponds to a narrow neuron-free zone containing only glial cells; this neuron-free zone is a normal variation (16) and should not be confused with focal HS. The external (superficial) edge of the pyramidal cell layer typically has an undulating shape in the subiculum proper that is absent or less prominent in the CA1. In addition, the external pyramidal cells are smaller and more clustered in the subiculum proper compared with those in the CA1; these small neurons break out to form spherical islands (layer II) in the distal part of the subiculum (presubiculum).

We did not include the presubiculum in the study because it is much less commonly involved by HS than the included regions. In addition, including more regions would have weakened the statistical analysis.

We use the term "CA4" for the pyramidal cells that are enclosed by the dentate gyrus, which is consistent with the use of this term by Lorente de No in some of his illustrations; however, in other illustrations, he applied the term "CA4" to the polymorphic layer of the dentate gyrus $(15,16)$.

\section{Immunohistochemistry}

Formalin-fixed, paraffin-embedded blocks of autopsy brain tissue (4 Km thick) were deparaffinized. Immunohistochemistry for ZNT3 was performed in some cases to define the border between the $\mathrm{CA} 3$ and the $\mathrm{CA} 2$. For antigen retrieval, the sections were placed in $\mathrm{CC} 1$ solution (Ventana Medical Systems, Tucson, AZ) and heated at $95^{\circ} \mathrm{C}$ for 30 minutes. The sections were exposed to a rabbit polyclonal antibody to human ZNT3 (BMP094; MBL Corp., Woburn, MA) at a dilution of 1:2000 for 32 minutes. The signal was developed with the ultraView Universal DAB Detection system (Ventana Medical Systems). The immunostaining procedure was performed using the Benchmark Ultra automated stainer (Ventana Medical Systems).

Immunohistochemistry for choline acetyltransferase (ChAT) was performed in some cases to delineate the border between the CA2 and the CA1. For antigen retrieval, the sections were placed in a Pascal programmable pressure cooker (Dako, Carpinteria, CA) containing Reveal Decloaker solution (Biocare Medical, Concord, CA), with the target temperature and time set to $125^{\circ} \mathrm{C}$ and 30 seconds, respectively. The sections were exposed to a rabbit polyclonal antibody to ChAT (AB143; Millipore, Billerica, MA) at a dilution of 1:400 for 32 minutes. The signal was developed with the Envision Plus Rabbit detection system (Dako). The immunostaining procedure was performed using the Autostainer Plus (Dako).

\section{Hirano Silver Staining}

Hirano silver staining was performed in some cases as an aid in defining the border between the CA1 and the subiculum. The staining was performed according to a published method (18). 


\section{Statistical Analysis}

The frequencies of involvement in the 3 diagnostic groups were compared using the Freeman-Halton extension of the Fisher exact probability test for 2-row $\times 3$-column contingency tables. A Web-based calculator was used to compute the 2-tailed $\mathrm{p}$ values (http://www.vassarstats.net/fisher2x3.html). The level of significance was set at $\mathrm{p}=0.05$.

\section{RESULTS}

Immunohistochemistry for ZNT3, which labels the hippocampal mossy fibers extending from the dentate to the CA3, worked consistently and was found to be helpful for defining the border between the CA3 and the CA2 (Fig. 2). Immunohistochemistry for ChAT was helpful in delineating the border between the CA2 and the CA1 because there is a drop in ChAT level that occurs at the border between the CA2 and the CA1. However, immunohistochemistry for ChAT did not work as consistently as that for ZNT3. The Hirano silver stain was helpful in some cases in defining the border between the CA1 and the subiculum by highlighting a bundle of axons that ends at this border, but this was not a consistent finding (Fig. 2).

The subiculum (either prosubiculum only or both prosubiculum and subiculum proper) was involved in $57 \%$ of HS-FTLD, $10 \%$ of H-I, and $0 \%$ of HS-TLE cases ( $<<0.0001$; Fig. 3). The CA regions other than CA1, most often CA4 and CA3, were involved in 57\% of HSTLE and $30 \%$ of H-I cases but never in HS-FTLD $(p=0.0003)$. Because there were cases where CA4 was involved without CA3 and vice versa, these percentages are higher than the numbers shown for the individual regions in Figure 3. The CA1-dist region was involved in $79 \%$ of HS-FTLD, $35 \%$ of $\mathrm{H}-\mathrm{I}$, and $37 \%$ of HS-TLE cases $(\mathrm{p}=0.02)$. The CA1-dist region was the only area involved in $29 \%$ of HS-FTLD, $3 \%$ of HS-TLE, and $0 \%$ of H-I cases ( $\mathrm{p}=$ $0.019)$. The CA1-prox region was the area most likely to be affected in $\mathrm{H}-\mathrm{I}(85 \%)$. The region extending from the CA1-prox to the CA1-mid was the only area involved in $50 \%$ of $\mathrm{H}-\mathrm{I}, 29 \%$ of HS-TLE, and $0 \%$ of HS-FTLD cases $(\mathrm{p}=0.004)$.

\section{DISCUSSION}

As expected, the CA1 region was the area most commonly involved by HS in HS-FTLD, HS-TLE, and H-I. Our study uncovered differences in the extent of involvement of the CA1-prox and the CA1-dist as well as the likelihood of involvement of hippocampal subregions other than the CA1 (Fig. 3). Although some of these findings, such as the involvement of the CA4-CA3 regions in a subset of TLE cases, have been established in previous literature $(1,2)$, to our knowledge, this is the first study where the anatomic extent of HS was directly compared among FTLD, TLE, and H-I cases in rigorously defined hippocampal subfields.

The novel findings included the observations that the CA1-dist and the prosubiculum were the areas that are most commonly involved in FTLD, whereas the CA1-prox and the CA1mid were more likely to be involved in TLE and H-I (Fig. 3). The area most commonly involved in ischemic injury is the CA1-prox (Fig. 3). Interestingly, the prosubiculum was occasionally involved even in the absence of CA1 involvement in FTLD; this finding 
underlines the specific vulnerability of the prosubiculum for HS in FTLD. A similar finding was previously reported in FTLD with motor neuron disease (FTLD-MND) by Nakano, who described depletion of neurons in the CA1-subiculum border zone in the rostral hippocampus (level of the pes hippocampi) (19). This lesion usually disappeared at more caudal levels of the hippocampus. In our series, 2 of the 3 FTLD-TDP cases with HS limited to the prosubiculum at the level of the lateral geniculate nucleus had no history of motor neuron disease (a detailed clinical history was unavailable in the third case), which suggests that the prosubiculum is highly vulnerable to HS in FTLD in general and not only in FTLDMND. In a post hoc analysis, we examined the prevalence of HS in the anterior hippocampus of our FTLD cases and found that HS was present in 50\% (21 out of 42) of FTLD cases in the anterior hippocampus compared with $31 \%$ (14 out of 45 ) in the posterior hippocampus ( $\mathrm{p}=0.08$, not significant). Hippocampal sclerosis limited to the prosubiculum occurred in $24 \%$ of the FTLD cases with HS in the anterior hippocampus and in $21 \%$ of the FTLD cases with HS in the posterior hippocampus (not significant). Because we only had 2 FTLD-MND cases, we could not determine the distribution and prevalence of HS in cases with MND versus cases without MND.

The differential vulnerability of the CA1-prox compared with the CA1-dist found in this study is surprising because CA1 is traditionally considered a single homogeneous subregion of the hippocampus. Further studies are needed to characterize the differences in neurochemistry and gene expression that might potentially underlie the differential vulnerability of the CA1-prox and the CA1-dist. The differences that have been uncovered to date include the finding that certain glial cell line-derived neurotrophic factor family ligands and receptors are preferentially expressed in the CA1-prox compared with the CA1dist $(20,21)$.

We found that labeling of the hippocampal mossy fibers by immunohistochemistry for ZNT3 was helpful in defining the border between CA3 and CA2 in routinely processed formalin-fixed human autopsy tissue (Fig. 2). Immunohistochemistry for ZNT3 has been previously used to stain the hippocampal mossy fibers in the mouse brain (22). The traditional method for staining the hippocampal mossy fibers is Timm stain for zinc, which requires treating the tissue with sulfide before fixation and is, therefore, unsuitable for routinely processed formalin-fixed human tissue (23).

We found that different hippocampal subfields and CA1 subsegments are preferentially involved in FTLD, TLE, and ischemic injury, suggesting that the pathogenesis of HS is at least to some extent different in these conditions. There is little doubt that release of glutamate in the extracellular space (excitotoxicity) plays an important role in hippocampal neuronal death in epilepsy. Although the distributions of glutamate receptor subtypes in the normal human hippocampus (24-26) do not appear to explain the selective vulnerability of the CA1-prox and the CA1-mid reported in the current study, this leaves open the possibility that the distributions of glutamate receptors could be altered in disease states compared with normal. Only limited studies addressing this possibility have been performed. In a receptor autoradiography study of the human hippocampus, highest binding for glutamate, NMDA, and AMPA receptors was seen in the molecular layer of the dentate gyrus and throughout CA1 in specimens of hippocampi from subjects without epilepsy, subjects with epilepsy 
caused by an extrahippocampal tumor, and subjects with TLE (26). Binding for kainate receptors was highest in CA3 (26).

As in TLE, there is strong evidence that excitotoxicity contributes to hippocampal neuronal death after a hypoxic insult. For example, a recent study showed a highly significant protection from hypoxic injury in all hippocampal subfields (CA1-CA4 and dentate gyrus) in GluK4 receptor-knockout mice (27). However, the precise mechanism mediating this widespread hippocampal neuroprotection is not clear because the expression of the GluK4 is concentrated in CA3 (27). In another animal experiment, a neonatal hypoxic insult resulted in an early (i.e. 6 hours after hypoxic insult) and nearly total loss of glial glutamate transporters throughout CA1, CA2, and CA3 but not in the dentate gyrus granule neurons, which corresponded to the areas of subsequent neuronal injury (28). The connection of these results to the more restricted areas of selective vulnerability in the adult human hippocampus, as observed in our study, is unclear.

To our knowledge, the distribution of glutamate receptor subtypes has not been studied in FTLD, which leaves open the possibility that altered distribution of these receptors could potentially explain the selective vulnerability of the CA1-dist and prosubiculum to HS in FTLD. In addition to excitotoxicity, alternative promising hypotheses on hippocampal selective vulnerability in ischemic injury and neurodegenerative diseases include impairment of protein degradation secondary to proteasomal stress (29).

Interestingly, the prosubiculum, a region that we found to be selectively vulnerable to HS in FTLD, is characterized by a unique neurochemical and gene expression profile as well as dense amygdalo-hippocampal terminal projections (17). These projections to the prosubiculum originate in the basal nucleus of the amygdala, express acetylcholinesterase, and do not significantly extend to the adjacent subiculum proper or CA1 (17). Future studies should look for a possible correlation between neuropathologic alterations such as TDP-43 inclusions in the basal nucleus of the amygdala and HS involving the prosubiculum in FTLD.

\section{Acknowledgments}

We thank Ping Shang for expert technical assistance. Niccole Williams and Agatha Villegas provided outstanding administrative professional services.

Supported by National Institutes of Health grants P30AG12300 and R01NS062080.

\section{REFERENCES}

1. Kim JH. Pathology of epilepsy. Exp Mol Pathol. 2001; 70:345-367. [PubMed: 11418013]

2. Thom M, Liagkouras I, Elliot KJ, et al. Reliability of patterns of hippocampal sclerosis as predictors of postsurgical outcome. Epilepsia. 2010; 51:1801-1808. [PubMed: 20738385]

3. Miyata H, Hori T, Vinters HV. Surgical pathology of epilepsy-associated non-neoplastic cerebral lesions: A brief introduction with special reference to hippocampal sclerosis and focal cortical dysplasia. Neuropathology. 2013; 33:442-458. [PubMed: 23530853]

4. Leifer D, Kowall NW. Immunohistochemical patterns of selective cellular vulnerability in human cerebral ischemia. J Neurol Sci. 1993; 119:217-228. [PubMed: 8277338] 
5. Nelson PT, Schmitt FA, Lin Y, et al. Hippocampal sclerosis in advanced age: Clinical and pathological features. Brain. 2011; 134:1506-1518. [PubMed: 21596774]

6. Taraszewska A, Zelman IB, Ogonowska W, et al. The pattern of irreversible brain changes after cardiac arrest in humans. Folia Neuropathol. 2002; 40:133-141. [PubMed: 12572919]

7. Stanika RI, Winters CA, Pivovarova NB, et al. Differential NMDA receptor-dependent calcium loading and mitochondrial dysfunction in CA1 vs. CA3 hippocampal neurons. Neurobiol Dis. 2010; 37:403-411. [PubMed: 19879359]

8. Butler TR, Self RL, Smith KJ, et al. Selective vulnerability of hippocampal cornu ammonis 1 pyramidal cells to excitotoxic insult is associated with the expression of polyamine-sensitive $\mathrm{N}$ methyl-d-aspartate-type glutamate receptors. Neuroscience. 2010; 165:525-534. [PubMed: 19837138]

9. Lewis KA, Su Y, Jou O, et al. Abnormal neurites containing C-terminally truncated alpha-synuclein are present in Alzheimer's disease without conventional Lewy body pathology. Am J Pathol. 2010; 177:3037-3050. [PubMed: 21056999]

10. Bonner HP, Concannon CG, Bonner C, et al. Differential expression patterns of Puma and Hsp70 following proteasomal stress in the hippocampus are key determinants of neuronal vulnerability. $\mathrm{J}$ Neurochem. 2010; 114:606-616. [PubMed: 20477911]

11. Pao WC, Dickson DW, Crook JE, et al. Hippocampal sclerosis in the elderly: Genetic and pathologic findings, some mimicking Alzheimer disease clinically. Alzheimer Dis Assoc Disord. 2011; 25:364-368. [PubMed: 21346515]

12. Walker MA, Highley JR, Esiri MM, et al. Estimated neuronal populations and volumes of the hippocampus and its subfields in schizophrenia. Am J Psychiatry. 2002; 159:821-828. [PubMed: 11986137]

13. West MJ, Gundersen HJ. Unbiased stereological estimation of the number of neurons in the human hippocampus. J Comp Neurol. 1990; 296:1-22. [PubMed: 2358525]

14. Rosene DL, Van Hoesen GW. Hippocampal efferents reach widespread areas of cerebral cortex and amygdala in the rhesus monkey. Science. 1977; 198:315-317. [PubMed: 410102]

15. Lorente de No R. Studies on the structure of the cerebral cortex II: Continuation of the study of the Ammonic system. J Psychol Neurol. 1934; 45:113-177.

16. Insausti, R.; Amaral, DG. Hippocampal formation. In: Paxinos, G.; Mai, J., editors. The Human Nervous System. 2nd ed.. Amsterdam, Netherlands: Elsevier; 2004. p. 871-914.

17. Ding SL. Comparative anatomy of the prosubiculum, subiculum, presubiculum, postsubiculum and parasubiculum in human, monkey and rodent. J Comp Neurol. 2013; 521:4145-4162. [PubMed: 23839777]

18. Yamamoto T, Hirano A. A comparative study of modified Bielschowsky, Bodian and thioflavin S stains on Alzheimer's neurofibrillary tangles. Neuropathol Appl Neurobiol. 1986; 12:3-9. [PubMed: 2422580]

19. Nakano I. Frontotemporal dementia with motor neuron disease (amyotrophic lateral sclerosis with dementia). Neuropathology. 2000; 20:68-75. [PubMed: 10935441]

20. Serra MP, Quartu M, Mascia F, et al. Ret, GFRalpha-1, GFRalpha-2 and GFRalpha-3 receptors in the human hippocampus and fascia dentata. Int J Dev Neurosci. 2005; 23:425-438. [PubMed: 16002253]

21. Pina Serra M, Quartu M, Ambu R, et al. Immunohistochemical localization of GDNF in the human hippocampal formation from prenatal life to adulthood. Brain Res. 2002; 928:138-146. [PubMed: 11844480]

22. Chi ZH, Wang X, Cai JQ, et al. Zinc transporter 3 immunohistochemical tracing of sprouting mossy fibres. Neurochem Int. 2008; 52:1305-1309. [PubMed: 18406010]

23. Danscher G, Zimmer J. An improved Timm sulphide silver method for light and electron microscopic localization of heavy metals in biological tissues. Histochemistry. 1978; 55:27-40. [PubMed: 76622]

24. Johnson M, Perry RH, Piggott MA, et al. Glutamate receptor binding in the human hippocampus and adjacent cortex during development and aging. Neurobiol Aging. 1996; 17:639-651. [PubMed: 8832639] 
25. Blumcke I, Behle K, Malitschek B, et al. Immunohistochemical distribution of metabotropic glutamate receptor subtypes mGluR1b, mGluR2/3, mGluR4a and mGluR5 in human hippocampus. Brain Res. 1996; 736:217-226. [PubMed: 8930327]

26. Brines ML, Sundaresan S, Spencer DD, et al. Quantitative autoradiographic analysis of ionotropic glutamate receptor subtypes in human temporal lobe epilepsy: Up-regulation in reorganized epileptogenic hippocampus. Eur J Neurosci. 1997; 9:2035-2044. [PubMed: 9421164]

27. Lowry ER, Kruyer A, Norris EH, et al. The GluK4 kainate receptor subunit regulates memory, mood, and excitotoxic neurodegeneration. Neuroscience. 2013; 235:215-225. [PubMed: 23357115]

28. Pow DV, Naidoo T, Lingwood BE, et al. Loss of glial glutamate transporters and induction of neuronal expression of GLT-1B in the hypoxic neonatal pig brain. Brain Res Dev Brain Res. 2004; 153:1-11.

29. Tsuchiya T, Bonner HP, Engel T, et al. Bcl-2 homology domain 3-only proteins Puma and Bim mediate the vulnerability of CA1 hippocampal neurons to proteasome inhibition in vivo. Eur J Neurosci. 2011; 33:401-408. [PubMed: 21198986] 


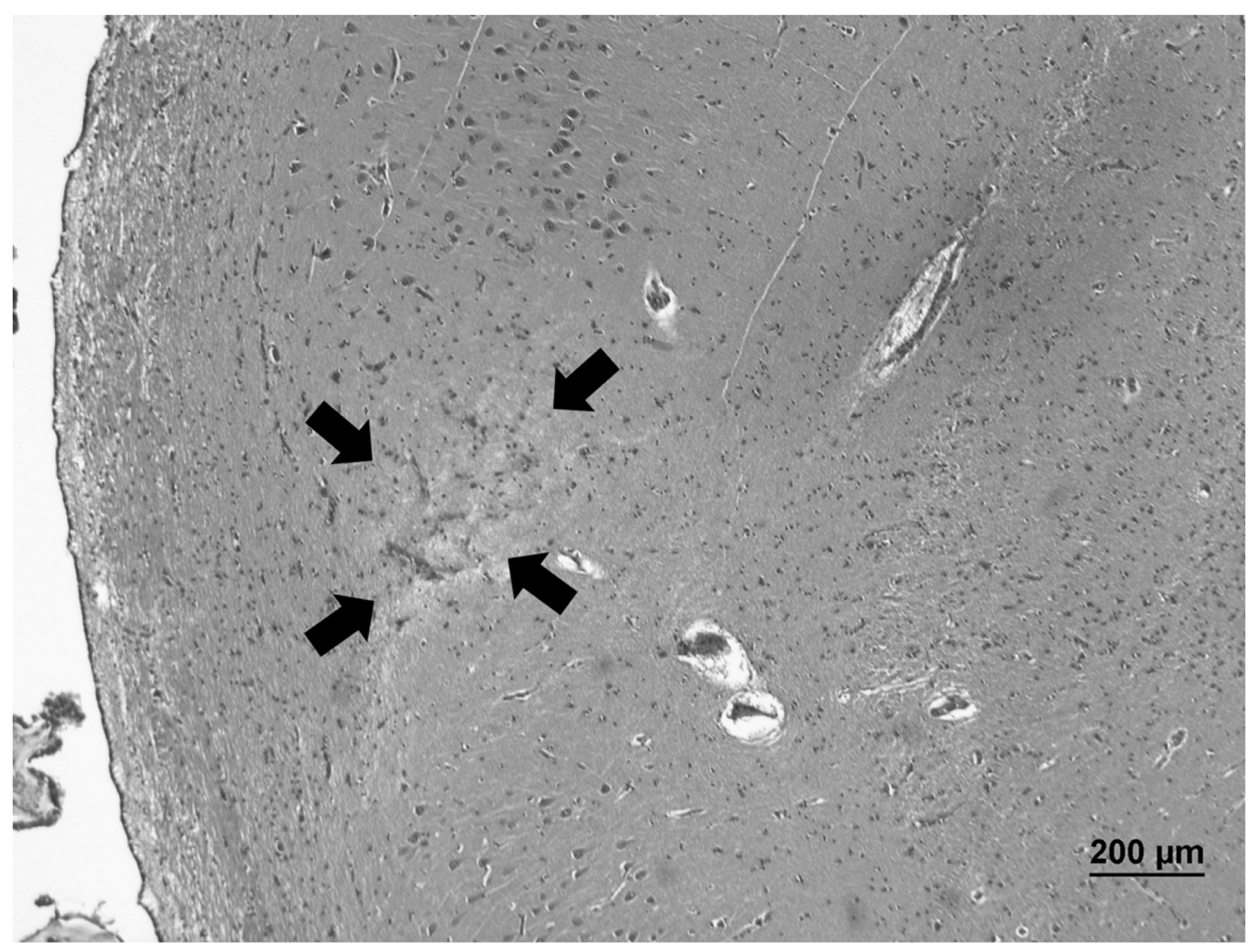

FIGURE 1.

An example of a case classified as hippocampal ischemic injury. A remote microinfarct (arrows) in the proximal CA1 of a subject with a clinical history of multiple strokes. Hematoxylin and eosin; original magnification: $40 \times$. Scale bar $=200 \mu \mathrm{m}$. 


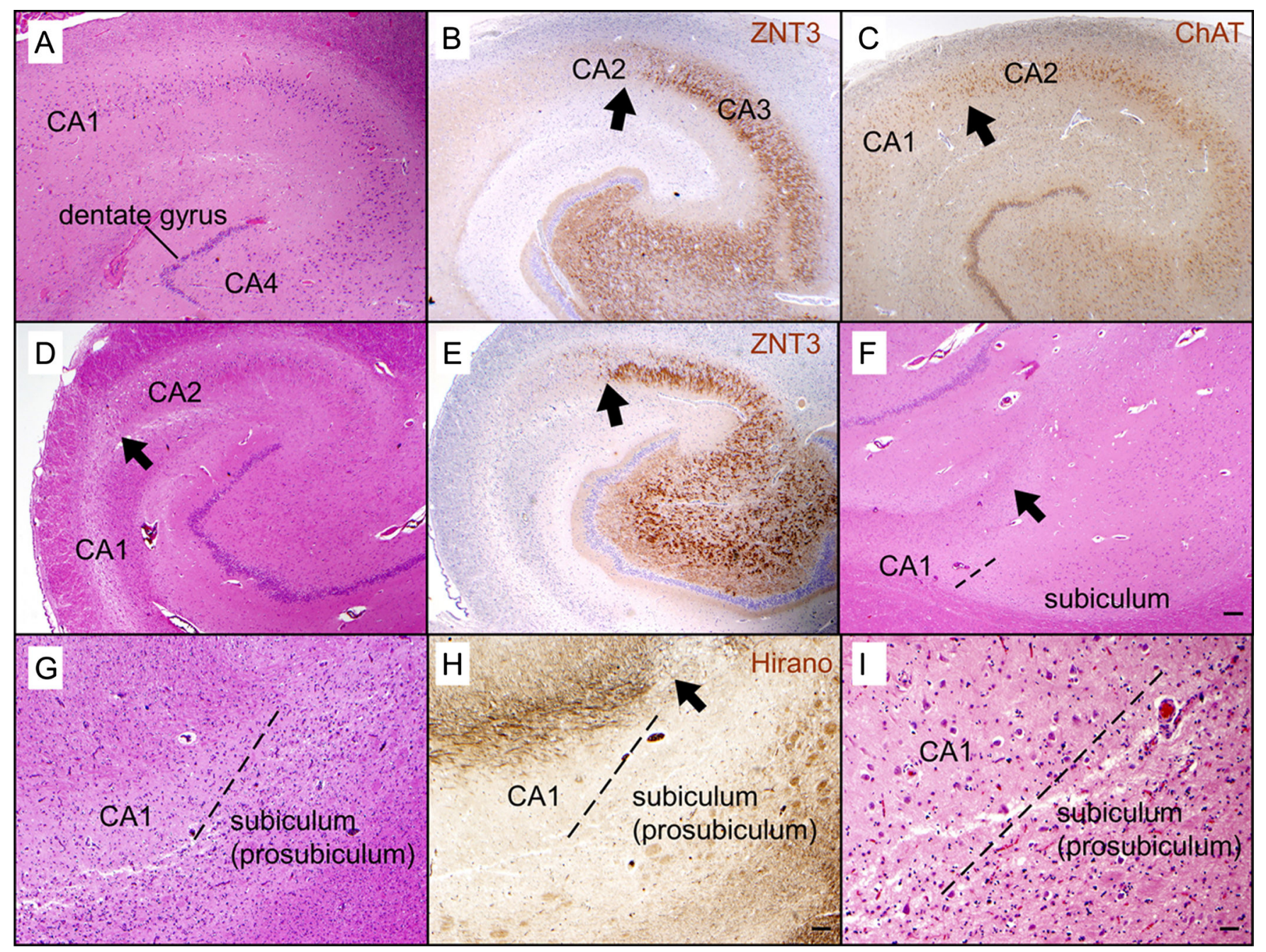

FIGURE 2.

Immunohistochemical and special stains can be helpful in delineating the hippocampal subfields in some cases. (A-C) Sections of the hippocampus without hippocampal sclerosis in a 90-year-old man. The border between CA2 and CA1 can be recognized based on the widening of the pyramidal cell layer when moving from CA2 to CA1 (A). Immunohistochemistry for ZNT3 highlights the mossy fibers extending from the dentate gyrus to CA3 but not CA2 (B), thus delineating the border between CA3 and CA2 (arrow). Immunohistochemistry for choline acetyltransferase (ChAT) shows a decrease in staining from CA2 to CA1 (C), which confirms the location of the border between CA2 and CA1 (arrow). (D-F) Sections of the hippocampus with hippocampal sclerosis (mesial temporal sclerosis) in a 44-year-old man with a history of temporal lobe epilepsy. Severe loss of neurons is limited to CA1, whereas CA2 is spared ([D] H\&E). An arrow points to the border between CA2 and CA1 (D). Immunohistochemistry for ZNT3 highlights the border between CA3 and CA2 ([E] arrow). The subiculum is spared from severe neuronal loss ([F] H\&E), which makes the oblique border between CA1 and the subiculum (dashed line) stand out. In this case, the CA1/subiculum border coincides with the termination of a strip of white matter in the overlying stratum lacunosum-moleculare (arrow); this strip may contain the Schaffer 
collaterals that extend from CA3 to CA1 but not subiculum. (G-I) Hippocampus of a 79year-old woman with frontotemporal lobar degeneration with TDP-43-positive inclusions (FTLD-TDP); the prosubiculum is severely affected by neuronal loss and gliosis, whereas CA1 and the subiculum proper are relatively spared ([G] H\&E). The border between CA1 and the prosubiculum is indicated by a dashed line $(\mathbf{G})$. A Hirano silver stain highlights a bundle of axons in the overlying stratum lacunosum-moleculare $(\mathbf{H})$; the bundle of axons terminates at the CA1/prosubiculum border (arrow), suggesting that some of the axons represent Schaffer collaterals. A higher magnification view of the CA1/prosubiculum border ([I] dashed line) shows relative sparing of neurons in CA1, which contrasts with the severe neuronal loss and gliosis in the prosubiculum. Original magnification: (A-F) 20x; (G-H) 40×; (I) 100x. Scale bars = (F) $200 \mu \mathrm{m}$ in (A-F); (H) $100 \mu \mathrm{m}$ in (G, H); (I) $40 \mu \mathrm{m}$. 


\section{Likelihood of involvement by HS}

\section{$70-100 \%$ \\ $50-69 \%$ \\ $30-49 \%$ \\ $10-30 \%$ \\ $0-10 \%$}
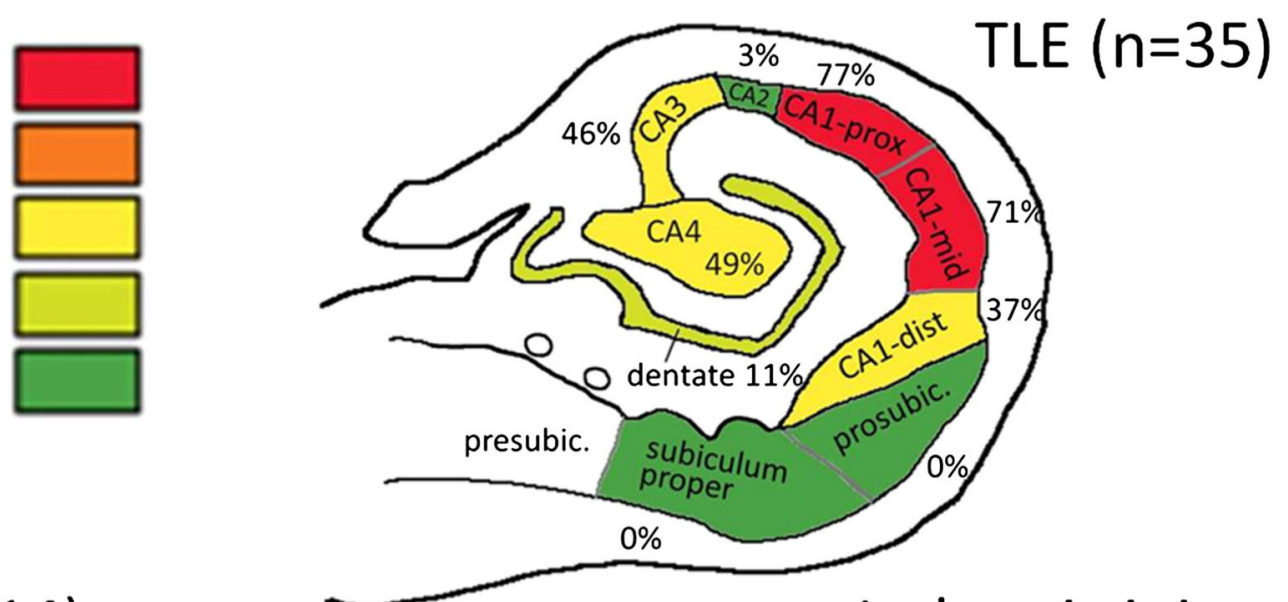

$\operatorname{FTLD}(n=14)$

\section{Ischemic injury $(n=20)$}
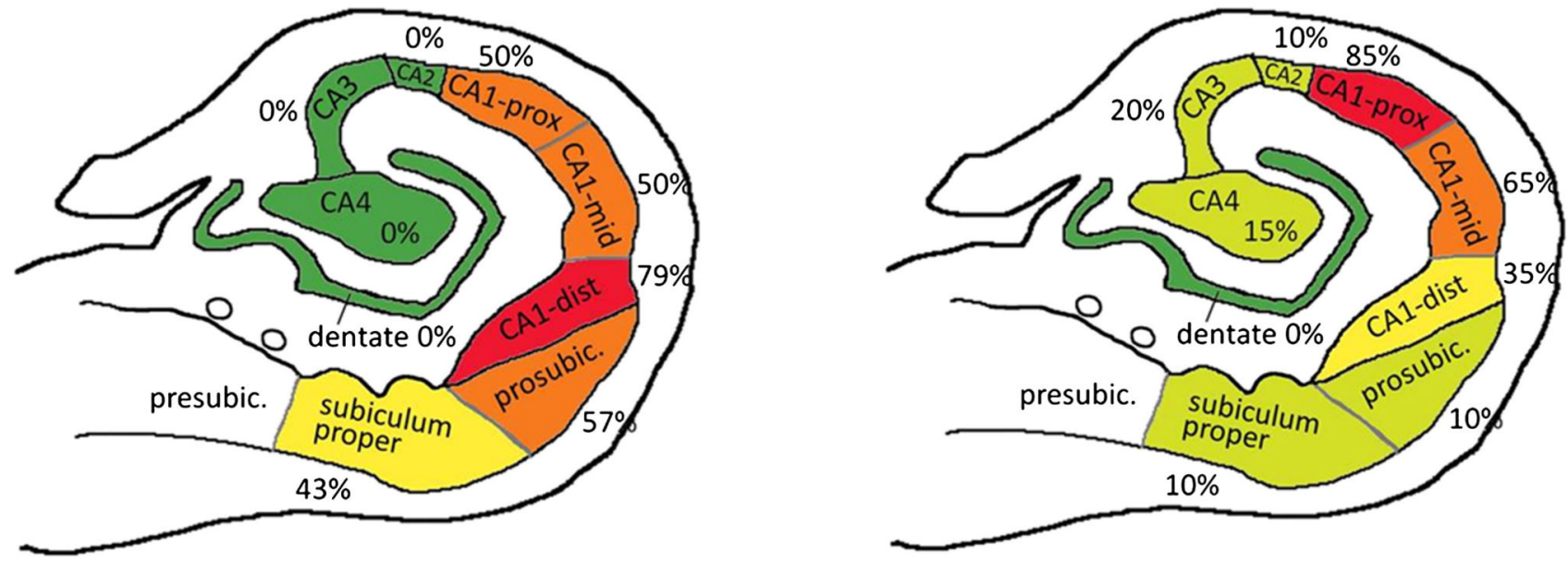

FIGURE 3.

Heat map showing the likelihood of involvement of hippocampal subfields by hippocampal sclerosis (HS), defined as severe neuronal loss, in frontotemporal lobar degeneration with HS (HS-FTLD), temporal lobe epilepsy with HS (HS-TLE), and hippocampal ischemic injury (H-I). HS-FTLD is distinguished from the other two groups by a higher likelihood of involvement of the CA1-dist and the subiculum. In both HS-TLE and H-I, the region extending from the CA1-prox to the CA1-mid was the area most commonly affected. Involvement of CA3 and CA4 was also common in HS-TLE but relatively infrequent in $\mathrm{H}-$ I. See Results for p values. dist, distal; presubic, presubiculum; prosubic, prosubiculum; prox, proximal. 